\title{
LXR regulate cholesterol homeostasis in the proximal mouse epididymis
}

\author{
Aurélia Ouvrier¹, Rémi Cadet¹, Jean-Marc A. Lobaccaro², Joël R. Drevet ${ }^{1 *}$ and \\ Fabrice Saez ${ }^{1}$
}

Université Blaise Pascal, UMR GReD CNRS 6247, INSERM U931

${ }^{1}$ Team "epididymis and sperm maturation", ${ }^{2}$ Team "LXR, oxysterols and steroidogenic tissues", 24 avenue des Landais, 63177 AUBIERE cedex, FRANCE

\begin{abstract}
Oxysterol nuclear receptors liver X receptors (LXR $\alpha$ and LXR $\beta$ ) regulate lipid homeostasis when cells have to face high amounts of cholesterol and/or fatty acids. Male mice invalidated for both $l x r$ (LXR-/-) are infertile by 5 months of age, and become sterile by the age of 9 months. The epididymis was previously shown to be affected by the gene invalidation, a phenotype specifically located in the two proximal segments of this organ. We demonstrate here that cholesteryl esters are accumulated in a specific cell type of the epididymal epithelium, the apical cells, in these two first segments, in LXR-/- male mice. These accumulations are correlated to a decrease in the amount of a specific membrane cholesterol transporter, ATP-binding cassette A1 (ABCA1) in the caput epididymidis of LXR-/- mice. This decrease is due to a transcriptional down-regulation, and we further demonstrate that ABCA1, in the two first segments of the caput epididymidis, is located in the apical cells, and that its accumulation is lost in these cells for LXR-/- male mice as soon as 4 months of age. These data bring new elements in the cholesterol trafficking pathways in the epididymis, and will help a better understanding of the molecular mechanisms occurring in this organ in relation to the sperm cells maturation process.
\end{abstract}

Key words: ABCA1, Liver X Receptor, apical cells, male fertility

\section{Introduction}

Liver X receptors (LXR) are nuclear receptors for oxysterols, metabolic derivatives of cholesterol, and are responsible, among other functions, for fatty acid synthesis and cholesterol homeostasis regulation [1]. LXR are present as two isoforms: $\operatorname{LXR} \beta(\mathrm{NR} 1 \mathrm{H} 3)$ which is expressed in tissues having an important lipid metabolism (testis and liver for example), and LXR $\alpha$ (NR1H2) which has a rather ubiquitous expression. These nuclear receptors form obligate heterodimers with retinoid $\mathrm{X}$ receptors (RXR), binding to a DR4 type of sequence in the promoter region of their target genes. When no ligands are available, RXR/LXR heterodimers repress gene expression via interaction with co-repressors and, upon binding of their ligands, co-activators are recruited and gene expression stimulated $[2,3]$

The development of 1xr-knockout (LXR-/-) mice

Correspondence: J.R. Drevet, CNRS UMR 6247/INSERM U

931, Clermont Université GReD, 24, avenue des Landais 63177 Aubičre cedex, France, Tel: (33) 4.73.40.74.13

Fax: (33) 4.73.40.52.45, e-mail : joel.drevet@univ-bpclermont.fr has helped to elucidate the roles of LXR in various tissues [4]. LXR-/- male mice have reduced fertility starting at 5 months of age, leading to sterility for nine month-old animals. The sterility phenotype is due to testicular defects, mainly lipid accumulation in the Sertoli cells and a lower proliferation rate of the germ cells [5]. These observations correlate with those of Robertson et al. [6] showing that LXR-beta is essential in retaining cholesterol homeostasis in Sertoli cells. The testicular phenotype is associated with a segment specific caput epididymidis dysfunction. Indeed, proximal caput segments 1 and 2 of these male mice show an enlargement of the tubule, the presence of an amorphous substance in the lumen and shrinkage of the epithelium height [7]. The sperm cells are affected and show midpiece fragility indicated by numerous broken cells, with a lot of isolated heads and flagella in spermatozoa recovered from LXR-/- mice cauda epididymides (mice of 8 months of age and older).

During epididymal transit, mammalian sperm cells undergo changes in membrane and cellular cholesterol contents [8] that are necessary for the further steps of the 
fertilization process. Molecular mechanisms regulating these transformations inside the epididymis are however poorly understood. Considering the well-known importance of LXR in cholesterol homeostasis regulation and the fact that lipid accumulations was previously shown by our group in the caput epididymidis of LXR-/- male mice [9], this study was aimed at further understanding the physiological implication of LXR in the caput epididymidis.

\section{Material and methods}

Animals. LXR-/- mice of the BL6 $\times 129$ Svj hybrid strain were housed under a $12 \mathrm{~h}$ light: $12 \mathrm{~h}$ darkness cycle in a temperature controlled $\left(22^{\circ} \mathrm{C}\right)$ atmosphere. Mice were handled according to the Guidelines on the Use of Living Animals in Scientific Investigations. Animals were fed a regular diet (2016 Teklad Global 16\% protein rodent diet, Harlan, France), and the western-diet consisted in a cholesterol enrichment up to $1.25 \%$.

Lipid staining. Lipid staining was performed on 8-?m-thick cryosections with oil red O (Sigma-Aldrich, Saint-Quentin Fallavier, France) for $4 \mathrm{~min}$ as previously described (Frenoux et al., 2004). Alternatively, cryosections were stained with Nile red $(2 \mu \mathrm{g} / \mathrm{ml}$ in PBS, Sigma), then $8 \mu \mathrm{m}$-thick cryosections counterstained with Hoechst 33342 solution $(1 \mu \mathrm{g} / \mathrm{ml}$, Sigma) and mounted with coverslips using PBS-glycerol (v/v) as mounting medium.

High Performance Thin Layer Chromatography (HPTLC). Epididymal lipids were extracted by the Folch method, with modifications [10]. Three different caput epididymides issued from three different animals were analyzed for each genotype. Cholesterol and cholesteryl esters were separated by HPTLC. The chromatograms were scanned, and spots were quantified via densitometry (Quantity One; Bio-Rad, Marnes-la-Coquette, France) by reference to different concentrations of standards on each HPTLC migration. Standard dosage values gave curves with linear-regression coefficients $\left(\mathrm{R}^{2}\right)$ of 0.90 or greater.

Western-blots and densitometry analysis. Proteins $(40 \mu \mathrm{g})$ were separated by SDS-PAGE and transferred onto nitrocellulose membrane (Hybond ECL, Amersham Biosciences, France). Blots were blocked with $10 \%$ low fat dried milk/0.1\% Tween 20/Tris Base Salt and then incubated with either anti- $\beta$ actin $(1 / 5000$, Sigma), antiABCA1 (1/500, Novus Biologicals, Littleton, USA) or anti-ABCG1 (1/1000, Novus Biologicals). Detection was performed with goat anti-rabbit horseradish peroxidase-conjugated secondary antibodies (1/5000, Amersham) using the "ECL Western Blotting Detection" kit (Amersham) on hyperfilms (Amersham). Densitometric analyses were carried out with "Quantity one" software (Bio-rad).

Quantitative RT-PCR. Total RNA was isolated using the Trizol method (Invitrogen, Cergy Pontoise, France) and reverse transcribed with Improm II Reverse Transcriptase (Promega, Charbonnieres, France) and random hexamer primers (Promega). Real-time polymerase chain reaction was performed on an iCycler (Biorad). Four?L of 1:50 diluted cDNA template were amplified using the qPCR assay kit, following the manufacturer's instructions (Biorad) using SYBR Green dye to measure duplex DNA formation. The sequences of primers used to amplify abcal are: fw-ggagctgggaagtcaacaac / rvacatgctctcttccegtcag; and for cyclophillin: fw-ggagatggcacaggaggaa / rv-geccgtagtgettcagett.

Immunofluorescence. After deparaffinization, $5 \mu \mathrm{m}$-thick sections were equilibrated 5min in PBS and blocked in PBS-1\% BSA
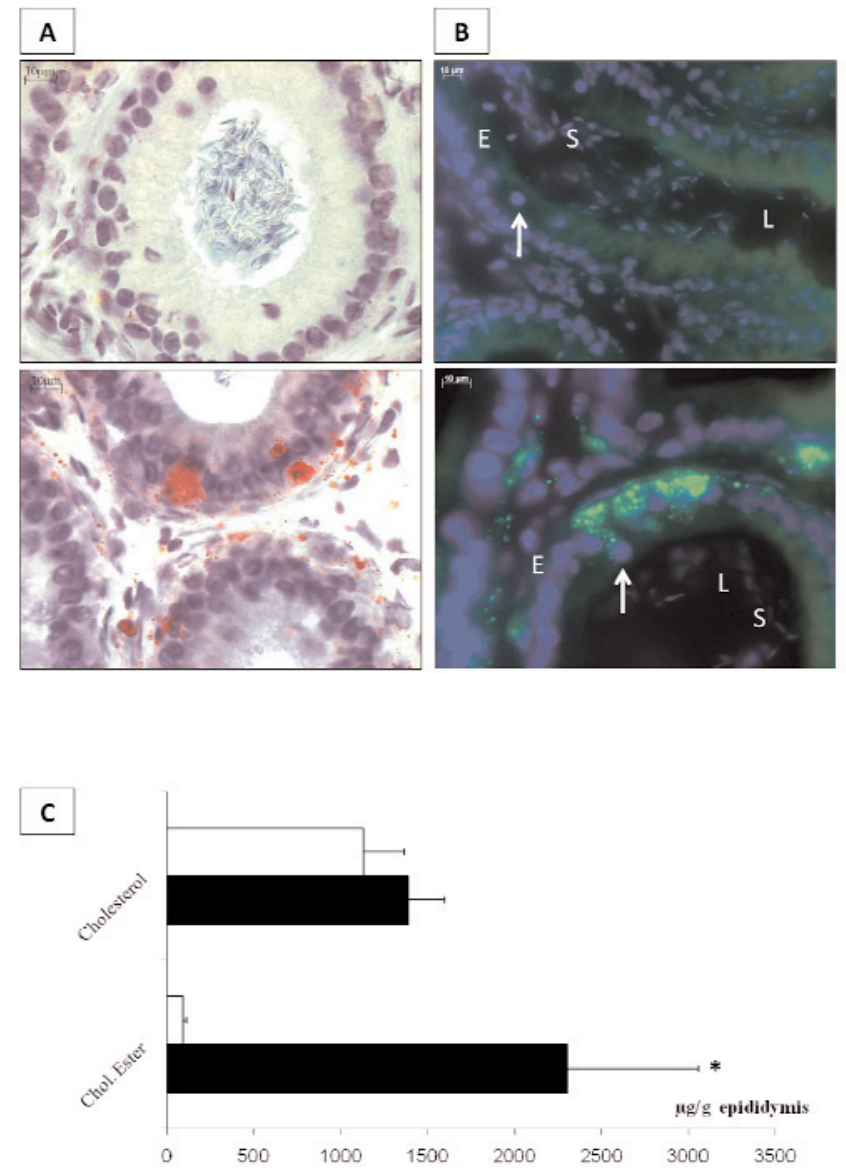

Fig. 1. Neutral lipid accumulations in caput epididymides of LXR/- mice. (A) Oil red O staining on cryosections from wild-type (upper picture) or LXR-/- (bottom picture) caput epididymidis at 4 months of age. Lipids appear in red. (B) Nile red staining on cryosections from wild-type (upper picture) or LXR-/- (bottom picture) caput epididymidis at 4 months of age. Green fluorescence indicates neutral lipid accumulations. $\mathrm{E}=$ epithelium, $\mathrm{L}=$ lumen, $\mathrm{S}=$ spermatozoa; arrows indicate apical cells as evidenced by their apical-located nuclei. (C) Densitometric analysis issued of the HPTLC assays allowing the quantification of cholesterol and cholesteryl esters. Bars are expressed as $\mu \mathrm{g}$ per mg of tissue extracted. ${ }^{*} \mathrm{p}<0.05$ compared to wild-type mice.

$(\mathrm{w} / \mathrm{v})-1 \%(\mathrm{w} / \mathrm{v})$ fetal calf serum, for $30 \mathrm{~min}$. Slides were incubated overnight at $4{ }^{\circ} \mathrm{C}$ with anti-ABCA1 antibody $(1 / 500$, Novus biological) diluted in PBS-0.1\% BSA (w/v). Sections were washed for $5 \mathrm{~min}$ in PBS, blocked for $20 \mathrm{~min}$ in PBS-1\%BSA and then incubated with FITC-conjugated secondary goat anti-rabbit Alexa 488 antibodies (1/1000 in PBS-0.1\%BSA, Invitrogen). Sections were counterstained with Hoechst 33342 solution $(1 \mu \mathrm{g} / \mathrm{ml}$, Sigma $)$ and mounted with coverslips using PBS-glycerol (v/v).

\section{Results}

Oil red O staining in LXR-/- mice showed lipid accumulations in cells surrounding the epididymal tubules, i.e. smooth muscle cells as well as in the epithelium (Fig. 1A). Nile Red staining on caput epididymidis sections of 4-month-old LXR-/- mice confirmed the presence of neutral lipids in smooth muscle cells and 

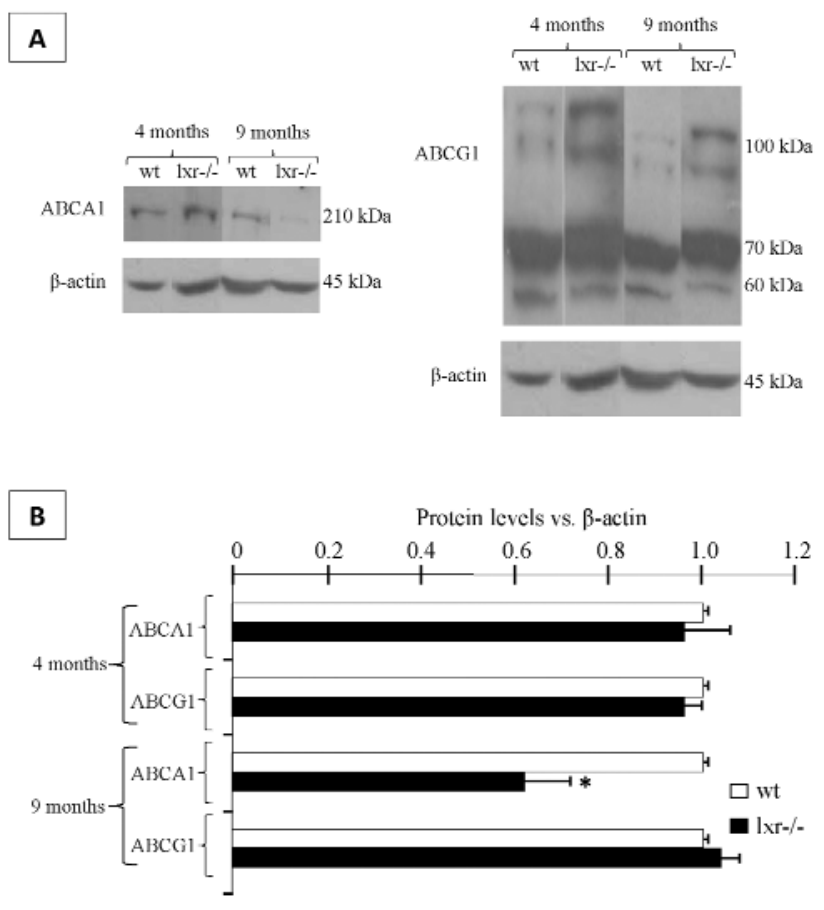

C

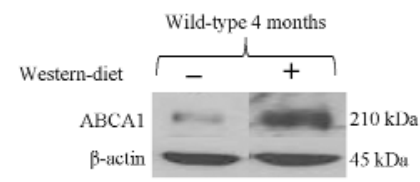

Fig. 2. ABCA1 is decreased in 9-month old LXR-/- animals. (A) Western-blots showing ABCA1, ABCG1 and beta-actin accumulations in caput epididymides of 4- and 9-month-old wild-type and LXR-/- mice. Each picture is representative of three different experiments made on 3 different animals. (B) Relative ABCA1 and ABCG1 protein levels in caput epididymidis in 4- and 9month-old wild-type and LXR-/- mice. Bars are expressed as mean \pm SEM of 3 different experiments using $\beta$-actin for relative quantification made on 3 different animals. ${ }^{*} \mathrm{p}<0.05$ compared to wildtype mice. (C) Western-blot showing ABCA1 protein levels in caput epididymidis in 8 month-old wild-type mice fed during 7 weeks with a western $\operatorname{diet}(1.25 \%$ cholesterol).

also revealed the presence of lipid droplets in one particular subtype of epithelial cells: the apical cells (Fig.1B), showing a nucleus in an apical position, near the epididymal lumen (arrow). The neutral lipids staining in apical cells were limited to segments 1 and 2 of the caput epididymides (data not shown).

HPTLC assays allowed determining that the neutral lipids revealed by the two staining procedures were cholesteryl esters (Fig. 1C). They were present in a significantly higher amount in LXR-/- caput epididymides at four months of age compared to wildtype mice.

Two major transmembrane cholesterol transporters regulated by LXR are ATP-binding transporters A1 and G1 (ABCA1 and ABCG1 respectively). They were present in caput epididimydis and ABCA1 was

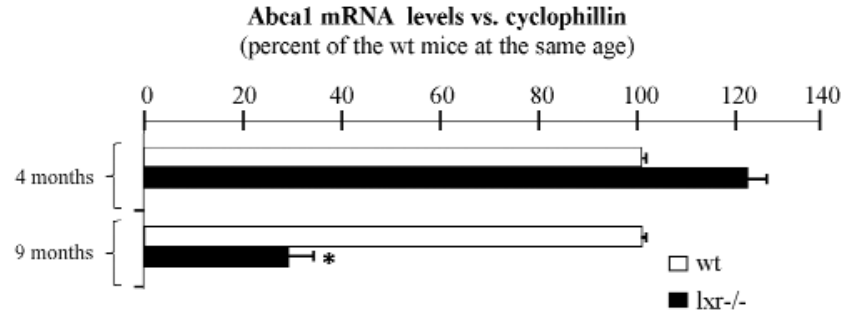

Fig. 3. abca1 is transcriptionally down-regulated in 9-month old LXR-/- animals. Relative expression level of abcal mRNAs in caput epididymides of 4- and 9-month-old wild-type and LXR-/mice. Bars are expressed as a percentage of the expression level in 1xr-/- animals vs an arbitrary $100 \%$ value in the wild-type animals. Each value is the mean $+/$ - SEM of 3 measurements performed on 3 different animals using cyclophillin as internal standard. $* \mathrm{p}<0.05$ compared to wild-type mice.

down-regulated at 9 months of age in LXR-/- animals compared to wild-type animals, whereas no change was seen at 4 months of age (Fig.2A and B). Concerning $\mathrm{ABCG} 1$, its accumulation was not modified depending on either the genotype or the age of the mice (Fig.2 A and B). To confirm the LXR-dependent regulation of $\mathrm{ABCA} 1$ in mouse caput epididymidis, we demonstrated that a cholesterol-enriched diet (known as western-diet) provoked a higher accumulation of ABCA1 in wild-type animals (Fig.2 C). This was not the case if 4 months-old LXR-/- mice were treated (data not shown) thus showing the dependence upon LXR for this regulation.

The lower accumulation of ABCA1 in 9 month-old animals was due to a transcriptional down-regulation as demonstrated in Fig. 3 where abcal mRNA showed a significant decrease in 9 month-old LXR-/- mice, whereas the expression levels were similar in wildtype and LXR-/- animals at 4 months.

ABCA1 immunofluorescence detection showed that this transporter was present, in wild-type mice epididymides, in the same type of epithelial cells in which we noticed neutral lipid accumulations in LXR-/- animals (see Fig.1A), the apical cells (Fig. 4A, arrows). This is the case in the two first caput segments, another point in correlation with the location of lipid accumulations (data not shown). At 4 months of age, the ABCA1 specific staining was lost in LXR-/- animals in the apical cells of the two first caput segments (Fig.4B, arrows).

\section{Discussion}

LXR are involved in cholesterol homeostasis in various organs, including male reproductive organs such as testis or other steroidogenic tissues [11]. This paper shows that these nuclear receptors also have a role to play in the epididymis. In this view, the apical cells of the two proximal caput segments seem to have 


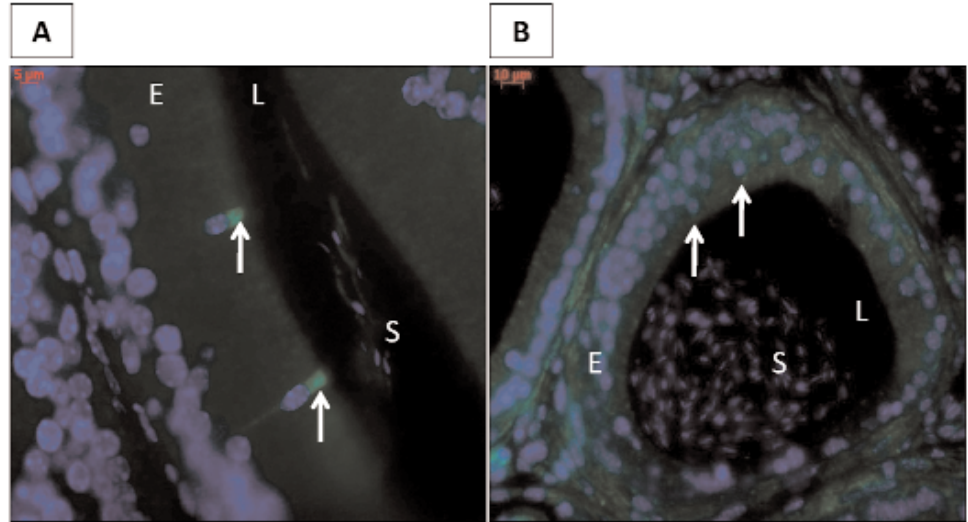

Fig. 4. ABCA1 is lost in the apical cells of the proximal epididymis. ABCA1 immunofluorescence staining in proximal caput epididymidis from wild-type (A) and LXR-/- (B) mice at 4 months of age. $E=$ epithelium, $L=$ lumen, $\mathrm{S}=$ spermatozoa; arrows indicate apical cells as evidenced by their apical-located nuclei. Each photomicrograph is representative of 3 different experiments made on 3 different individuals. a particular function. Indeed, cholesteryl ester accumulations in these cells were associated with the loss of ABCA1, an important protein for cellular cholesterol efflux. It is likely that the two first epididymal segments play a special role in cholesterol trafficking, because in the more distal caput segments (segments 3 to 5) ABCA1 staining was found in principal cells, a point in correlation with the recent data presented by Morales et al.[12], who also reported the detection of ABCA1 in the principal cells of mouse caput epididymidis. Interestingly, ABCA1 accumulation was lost in the segments where the epididymal phenotype, i.e. mainly epithelial shrinkage and tubule lumen enlargement, was previously described [7]. The link between these events will still have to be investigated. The molecular mechanisms underlying these modifications are probably intricate as we could also show that only ABCA1 was lost whereas ABCG1 was not disturbed by the $l x r$ invalidation, even if ABCG1 is also a known target of LXR in other tissues.

Cholesteryl ester accumulations were likely due the loss of the cells ability to efflux cholesterol from their cytoplasm via ABCA1, rather than perturbations of the intracellular metabolism (de novo synthesis, uptake and esterification) as the total amount of cholesterol was not modified (see Fig. 1B). This point needs to be confirmed by further analysis of the metabolic pathways involved, a work that is currently being investigated.

ABCA1 accumulation in apical cells was lost at 4 months of age whereas the total quantity of the protein at the same age was not changed (See Fig. 2, A and B). This discrepancy is related to the fact that westernblots were performed on whole caput extracts, and as we mentioned earlier, ABCA1 is present in the cytoplasm of principal cells in caput segments 3 to 5 . The sensitivity of the protein detection in the western-blot assays did not allow revealing the specific loss in apical cells. At 9 months of age, it is likely that ABCA1 is down-regulated in the whole caput, implicating a transcriptional regulation.
The presence of ABCA1 and ABCG1 in the epithelial cells of the epididymis suggest that cholesterol needs to reach the epididymal lumen, and probably interact with maturing sperm cells, as these two transporters are shown on the apical side of the epithelium. Indeed these proteins are specialized in cholesterol efflux $[13,14]$, which also suggest the presence of cholesterol acceptors in the lumen. This point is not easy to investigate as it is quite impossible to harvest luminal fluid from a mouse caput epididymidis due to the small size of the organ. However, these questions will have to be answered in the future to understand the exact role played by LXR in the sperm maturation processes.

In conclusion, this paper brings new insights in LXR-related cholesterol homeostasis and trafficking in the mouse caput epididymidis, and these data will be helpful to better understand the regulation of sperm plasma membrane maturation during their epididymal transit.

\section{References}

[ 1] Wójcicka G, Jamroz-Wiśniewska A, Horoszewicz K, Bełtowski1 J. Liver X receptors (LXRs). Part I: Structure, function, regulation of activity, and role in lipid metabolism. Postepy Hig Med Dosw. 2007;61:736-759.

[2] Ahuja HS, Szanto A, Nagy L, Davies PJ. The retinoid X receptor and its ligands: versatile regulators of metabolic function, cell differentiation and cell death. J. Biol. Regul. Homeost. Agents . 2003;17:29-45.

[3] Wagner BL, Valledor AF, Shao G, et al. Promoter-specific roles for liver $\mathrm{X}$ receptor/corepressor complexes in the regulation of ABCA1 and SREBP1 gene expression. Mol.Cell. Biol. 2003;23:5780-5789.

[4] Tontonoz P, Mangelsdorf DJ. Liver X receptor signaling pathways in cardiovascular disease. Mol Endocrinol. 2003; 17(6):985-93.

[ 5] Volle DH, Mouzat K, Duggavathi R, et al. Multiple roles of the nuclear receptors for oxysterols liver X receptor to maintain male fertility. Mol. Endocrinol. 2007;21:1014-1027.

[6] Robertson KM, Schuster GU, Steffensen KR. et al. The liver $\mathrm{X}$ receptor $\beta$ is essential for maintaining cholesterol homeostasis in the testis. Endocrinol, 2005;146:2519-2530. 
[7] Frenoux JM, Vernet P, Volle DH, et al. Nuclear oxysterol receptors, LXR, are involved in the maintenance of mouse caput epididymidis structure and functions. J Mol Endocrinol. 2004;33:361-375.

[ 8] Rejraji H, Sion B, Prensier et al. Lipid remodeling of murine epididymosomes and spermatozoa during epididymal maturation. Biol Reprod. 2006;74:1104-1113.

[ 9] Saez F, Chabory E, Cadet R, et al. Liver X receptors and epididymal epithelium physiology. Asian J Androl. 2007;9:574582.

[10] Force A, Grizard G, Giraud MN, Motta C, Sion B, Boucher D. Membrane fluidity and lipid content of human spermatozoa selected by swim-up method. Int J Androl. 2001;24(6): 327-34.
[11] Volle DH, Lobaccaro JM. Role of the nuclear receptors for oxysterols LXR in steroidogenic tissues: beyond the "foie gras", the steroids and sex? Mol Cell Endocrinol. 2007;265:183-189.

[12] Morales CR, Marat AL, Ni X, et al. ATP-binding cassette transporters ABCA1, ABCA7, and ABCG1 in mouse spermatozoa. Biochem Biophys Res Commun. 2008;376:472-477.

[13] Oram JF, Vaughan AM. ATP-Binding cassette cholesterol transporters and cardiovascular disease. Circ Res. 2006;99(10):1031-1043.

[14] Baldán A, Tarr P, Lee R, Edwards PA. ATP-binding cassette transporter G1 and lipid homeostasis. Curr Opin Lipidol. 2006;17(3):227-32. 